\title{
Reflexiones sobre los principios éticos en investigación biomédica en seres humanos
}

\author{
MARIANNE GAUDLITZ H.*
}

\section{Ethical principles in biomedical research involving human beings}

Biomedical research involving human subjects present valoric problems. A scientific research must go hand in hand with several requisites to be ethic. Human experimentation through its history in the occidental world has not been exempt from abuses. Ethic international rules have appeared after the transgressions. The protection of the human beings that freely and willingly participate in a biomedical research is an ethic obligation. The dignity of the human beings is above the progress of science and the wealth it carries with it.

Key words: Scientific progress, biomedical research, ethical requirements, dignity of the human beings, vulnerable populations, protection to subjects.

\section{Resumen}

La investigación biomédica en seres humanos plantea problemas valóricos. Una investigación científica debe ir de la mano de una serie de requisitos para que sea una investigación ética. La trayectoria de la investigación en el mundo occidental no ha estado libre de abusos. Las normas éticas internacionales han aparecido después de las transgresiones. La protección de los probandos que participan libre y voluntariamente en una investigación biomédica es una obligación ética, la dignidad de éstos está por sobre el progreso de la ciencia y el bienestar que éste acarrearía.

Palabras clave: Progreso científico, investigación biomédica, requisitos éticos, dignidad del ser humano, poblaciones vulnerables, protección de probandos.

\section{Introducción}

Involucrar a seres humanos en investigación biomédica es un tema complejo. También es un tema de valores. El dilema se ubica entre el valor del progreso de la ciencia y el valor de la protección a los seres humanos que participan voluntariamente como probandos. ¿Podemos privilegiar el progreso de la medicina o de la ciencia por sobre el ser humano y nuestra obligación para con él? ¿Cómo encontrar el equilibrio justo? Éticamente la investigación en seres humanos puede justificarse aduciendo el argumento del bien común, entendido en el sentido de progreso. La investigación biomédica tiene como expectativas encontrar nuevas formas de bene- ficiar la salud y el bienestar de las personas y, ofrece encontrar un conocimiento que de otra forma no podría obtenerse. No debemos olvidar que los sujetos participantes en investigación son sólo un medio para lograr este conocimiento y corren el riesgo de ser perjudicados en aras del bien de otros.

\section{Breve reseña histórica}

Hasta el siglo XX, la trayectoria de la investigación científica en seres humanos fue errática y sin control ético alguno, excepto la conciencia moral de los investigadores. Tradicionalmente la investigación clínica se hizo con sujetos sanos, * Departamento de Bioética y Humanidades Médicas, Vicepresidente Comité de Ética de Investigación en Seres
Humanos. Facultad de Medicina, Universidad de Chile. 
la investigación en sujetos enfermos se consideraba parte del tratamiento y no llegó nunca a plantear problemas éticos ${ }^{1}$. El consentimiento voluntario que comenzó a implementarse alrededor de 1930, puso en primer plano el criterio ético de autonomía por sobre el criterio de beneficencia con lo cual se favoreció la aparición de abusos. Fueron los abusos en la experimentación en los campos de concentración de la Alemania nazi los que dieron origen al Código de Nuremberg en 1947, cuyo principal postulado era la participación voluntaria e informada de todos los sujetos reclutados para una investigación. Sin embargo, este código se refiere sólo al respeto de la autonomía tanto de probandos como de investigadores y no hace referencia a la necesidad de legislar localmente. Según Diego Gracia, la idea de la autorregulación de los científicos, después de los horrores nazis, no se cumplió y los abusos continuaron y fueron haciéndose cada vez más frecuentes. En 1959, el médico estadounidense Henry Beecher publicó "Experimentation in Man", un libro que sería paradigmático, en el cual planteó insuficiencias en el Código de Nuremberg y propuso velar por el buen diseño de los experimentos y la calidad moral de los investigadores. Beecher fue el principal redactor del Código de Helsinski (1964) de la Asociación Médica Mundial que dividió la investigación médica en terapéutica y no terapéutica. A pesar de estos avances, algunos científicos denunciaron el carácter no ético de muchas investigaciones $\mathrm{y}$, los artículos que posteriormente escribió Beecher en relación a serias situaciones, sensibilizaron al público estadounidense. Se conocieron por la prensa algunas investigaciones que fueron haciéndose emblemáticas como el caso del Hospital Judío de Enfermedades Crónicas de Brooklyn (1963) en que se inyectó por vía subcutánea células cancerosas a 22 pacientes sin su consentimiento, y el caso de la State School (Escuela Estatal) Willowbrook, asilo de niños retrasados mentales, en que se infectó deliberadamente de hepatitis a niños recién ingresados, con el argumento que igual iban a contraer la enfermedad en el establecimiento. No obstante las graves transgresiones éticas cometidas por los estudios ya citados, fue el Estudio de Sífilis Tuskegee el que causó mayor conmoción pública. Esta investigación comenzó en 1932, y su objetivo era analizar el curso natural de la sífilis por medio del seguimiento de 400 varones negros en Alabama. En este estudio no se intentó ofrecer una posibilidad terapéutica a los probandos, aunque la penicilina había sido descubierta en 1950. En 1972 el periódico "The
New York Times" publicó un artículo que informaba de estos hechos, lo cual dio origen al escándalo y a la suspensión del proyecto.

La alarma pública creada en los EE.UU. especialmente sensible a la segregación racial y a los derechos individuales, llevó al presidente de ese país a encargar al Congreso de la nación. la creación de la Comisión Nacional para la Protección de los Sujetos Humanos en la Investigación Biomédica (1974), la cual dio origen al Informe Belmont (1978). Este informe estableció tres principios éticos destinados a servir de ayuda a científicos, sujetos de investigación y ciudadanos interesados en comprender los conceptos éticos inherentes a la investigación con seres humanos. El principio de respeto por las personas radica en el derecho del paciente o del voluntario sano a decidir libre e informadamente su participación en un estudio y se traduce en el consentimiento informado y el respeto por la autonomía. El principio de beneficencia se traduce en los esfuerzos por promover el bien del otro y la responsabilidad primera de cuidar el bienestar del sujeto de investigación. Este principio vela por lograr el máximo de beneficio con el mínimo de riesgo y sobre todo no dañar. El principio de justicia se traduce por la selección equitativa de los probandos, un acceso equitativo a riesgos y beneficios. Se incluye en este principio que los beneficios derivados de la investigación deben ser aprovechados en primer lugar por los sujetos que participaron en la investigación.

\section{Regulaciones éticas internacionales}

Las normas éticas internacionales que regulan la investigación en seres humanos se caracterizan por la convocación a los organismos deliberantes ante la denuncia de prácticas inmorales de investigación, primero aparece la transgresión y enseguida la sugerencia de como evitarla. No es el propósito de este artículo hacer un análisis profundo de las regulaciones internacionales sino un somero resumen.

Terminada la segunda guerra mundial y ante el horror por los abusos del régimen nazi, en la investigación en seres humanos, se dictó el Código de Nuremberg (1947) que enfatizó la protección de la autonomía del sujeto por medio del consentimiento voluntario para su participación en una investigación. Secundariamente estableció la necesidad de una conducta ética de los investigadores.

La Declaración Universal de los Derechos 
Humanos aprobada por las Naciones Unidas en 1948, expresa el valor humano fundamental de la protección de los derechos y bienestar de todos los sujetos humanos en la experimentación científica.

La Declaración de Helsinski (1964) formulada por la Asociación Médica Mundial establece pautas éticas para los médicos involucrados en investigación biomédica, tanto clínica como no clínica y ha servido para establecer pautas de investigación internacionales, regionales o nacionales. Esta declaración ha sido revisada en varias ocasiones y se ha perfeccionado en relación a la inclusión en investigación biomédica de poblaciones vulnerables. Las pautas éticas internacionales para la investigación biomédica en seres humanos (CIOMS 1993) fueron revisadas y el nuevo texto de $2002^{2}$, está destinado a orientar, especialmente a los países de escasos recursos, en la definición de pautas nacionales sobre ética de la investigación biomédica, aplicando estándares éticos en condiciones locales.

\section{Requisitos para que una investigación biomédica sea ética}

Los requisitos para que una investigación biomédica sea ética están en primer término orientados a la protección y respeto a las personas que participan mientras éstos contribuyen al bien social. Ezekiel Emmanuel ${ }^{3}$, ha establecido que una investigación debe reunir una serie de requisitos para que cumpla con un estándar ético que asegure una protección a las personas que participan como voluntarios.

En primer lugar, la investigación debe tener valor ${ }^{3}$, es decir, aportar con mejoras a la salud y al bienestar o al conocimiento de la información de la población. Como ejemplo de investigación que no tiene valor son aquellos estudios que duplican innecesariamente una información ya obtenida, resultados aprobados o una hipótesis banal. Según Emmanuel, el valor social o científico es un requisito ético porque una investigación valiosa utiliza responsablemente los recursos disponibles y evita la explotación de los probandos. No debe exponerse a los seres humanos a riesgos o daños potenciales a menos que se espere un resultado valioso. Al evaluar si un protocolo de investigación clínica es ético, debemos ocuparnos antes que nada de si tiene un valor social.

En segundo lugar, la validez científica es un principio ético en sí, ya que una investigación mal diseñada, con resultados poco confiables científicamente, no es ética. La Asociación Médica Mundial ha establecido que los investigadores deben asegurar que los estudios propuestos en seres humanos estén de acuerdo con principios científicos generalmente aceptados. La metodología debe ser válida, o sea, debe tener un objetivo científico claro que se pueda probar y los investigadores deben ser personas calificadas y con experiencia para llevar a cabo correctamente esta labor. Sin validez científica el estudio no genera conocimiento, no produce beneficio alguno y no justifica que se arriesgue o se dañe a las personas.

En tercer lugar, la selección equitativa de los sujetos que participarán en una investigación requiere que ningún grupo o clase de personas soporte una carga superior a la que corresponde a una justa distribución. Una selección de sujetos puede considerarse equitativa cuando éstos estén en condiciones de beneficiarse si la investigación tiene como resultado un tratamiento. Debe tomarse en cuenta que los resultados de la investigación tengan un valor real para los grupos que se reclutan para participar en la investigación. Los riesgos de participar deben reducirse al mínimo en cambio debe maximizarse los beneficios sociales de los resultados de la investigación. Es importante considerar que no se debe someter a riesgos a poblaciones vulnerables, como etnias estigmatizadas, presos o pobres, por el solo hecho que pudieran ser fácilmente reclutadas. La selección de sujetos debe ser lo más amplia posible dentro de lo que permite el objetivo de la investigación.

En cuarto lugar se sitúa la proporción riesgo beneficio $^{3}$. Al formular un proyecto de investigación en seres humanos es indispensable considerar que éste puede implicar tanto riesgos considerables como beneficios. La investigación sólo puede considerarse ética cuando los riesgos potenciales a los probandos se minimizan y los beneficios tanto para los individuos participantes como para la sociedad se maximizan. En las intervenciones en que se considera la posibilidad de un beneficio diagnóstico, terapéutico o preventivo para el sujeto reclutado debe considerarse que los beneficios para él serán al menos tan ventajosos como cualquier otra alternativa disponible ${ }^{4}$. Las consideraciones relativas al bienestar de los sujetos debieran tener prioridad sobre los intereses de la ciencia y la sociedad. Las intervenciones que no producen beneficios directos deben ser evaluadas en relación al conocimiento que se espera obtener. Los riesgos 
para grupos de personas, (grupos raciales, comunidades, etc) también deben ser considerados en virtud de que no sean discriminados o estigmatizados cuando se trata de investigaciones en campos como la genética o la epidemiología.

Evaluación independiente. La investigación clínica debe ser revisada por personas calificadas que no estén afiliados al estudio. Es una manera de reducir el impacto potencial que una investigación pueda tener sobre los participantes en un estudio. La revisión garantiza que los sujetos serán tratados éticamente y no sólo como un medio. El comité de evaluación ética es responsable de proteger los derechos, la seguridad y el bienestar de los sujetos de investigación. Un comité de evaluación ética debe ocuparse al mismo tiempo de la evaluación científica. Como hemos dicho anteriormente una investigación científicamente mal diseñada no es ética, la participación tanto de científicos como de probandos en un proyecto que no será válido significa una pérdida tanto de tiempo como de recursos. El comité debe por lo tanto contar con un grupo de expertos que verifique que la investigación es científicamente válida.

Como sexto punto ${ }^{3}$, el Consentimiento Informado asegura que los individuos que participen en una investigación están concientes de las condiciones en que participan, lo cual permite una decisión autónoma y el consiguiente respeto a la persona. En toda investigación biomédica que se realiza en seres humanos, el investigador debe obtener el consentimiento informado del potencial probando o en el caso de una persona incapaz de dar su consentimiento, la autorización de un representante legalmente calificado según las reglamentaciones locales. La revisión ética de un proyecto por un comité independiente garantiza al individuo que los resguardos tanto científicos como éticos serán cumplidos.

Por último, el respeto a los sujetos inscritos debe ser resguardado mientras dure la investigación. Este respeto permite al sujeto cambiar de opinión y retirarse de la investigación sin sanción, permite mantener su privacidad y se extiende también a proporcionar el tratamiento adecuado en caso de eventos adversos. Muchos investigadores tienden a considerar que el proceso concluye al inscribirse al individuo en el estudio. Sin embargo, no es así y es necesario recalcar que este proceso se extiende mientras dure la participación del sujeto. El seguimiento continuo vela por los intereses de los probandos y permite que éstos sean informados sobre la marcha de la investigación.

\section{Poblaciones vulnerables e investigación en países en desarrollo}

Se define "vulnerabilidad" como la condición mostrada por un individuo o grupo de personas cuando la capacidad de emitir juicios y tomar decisiones ha sido comprometida y no puede ejercerse, en virtud de alguna incapacidad o posición en la vida, lo que se traduce en incapacidad sustancial de proteger sus propios intere$\mathrm{ses}^{2}$. Investigar en un grupo o una población vulnerable plantea desafíos éticos adicionales y una preocupación especial por el grupo o individuos en que se planea efectuar la investigación. Se consideran cinco principales grupos vulnerables en la investigación médica: discapacitados en cuanto a su desarrollo, niños, ancianos, enfermos mentales, presos y poblaciones de minorías políticamente divididas o discriminadas. Mención especial tienen los grupos de prisioneros que llevan una vida altamente reglamentada con pérdida de la autonomía en que existe la posibilidad de pervertir el consentimiento libre, informado y voluntario necesario para participar y en los cuales la posibilidad de una coerción sutil está siempre presente. Los sujetos deben ser seleccionados por razones directamente relacionados con el problema en estudio y no debido a su fácil disponibilidad o a su posición o situación comprometida.

La investigación en países en desarrollo plantea ventajas, objeciones y exigencias para proyectos que tienen su origen en países desarrollados. Estos estudios tienen la ventaja que ofrecen a los países en desarrollo la introducción de nuevas tecnologías, capacitación del personal sanitario, mejoramiento de infraestructuras y tratamientos estandarizados y la formación de investigadores locales. Sin embargo, los investigadores locales no participarán en la elaboración ni en el análisis posterior de los proyectos.

Para los pacientes enrolados puede haber beneficios directos o indirectos, pero muchas veces los probandos participarán aportando al conocimiento sin llegar a gozar de los beneficios. El consentimiento informado redactado en el país de origen frecuentemente es poco comprensible y existe el riesgo de abuso. Debe exigirse un consentimiento informado redactado de acuerdo a la idiosincrasia local. Sin duda la investigación en países en desarrollo es más económica y ofrece menos dificultades, éstos deben exigir que los temas investigados sean de interés para el país: las revisiones realizadas por un comité independiente, deben asegurar las mismas exigencias que en el país de origen del proyecto; 
los estudios de fase 1 deben ser efectuados en el país de origen; debe haber compensaciones por riesgos; y se debe definir las compensaciones económicas.

\section{Conclusión}

La investigación biomédica que involucra a seres humanos es un tema complejo y se mueve entre el progreso de la ciencia y la protección a los seres humanos que participan en ella. Sin embargo, los límites de una investigación deben estar dados por el respeto a la dignidad de las personas. El fin último de una investigación biomédica debe ser el mismo que el fin de la medicina: ayudar a aliviar el sufrimiento de los enfermos. La regulación ética permite disminuir el riesgo de daño o atropello a la dignidad de las personas. La responsabilidad profesional de los investigadores y de las instituciones participantes es la base para proteger efectivamente a los probandos.

\section{Bibliografía}

1 .- GRACIA GUILLÉN D. Investigación en sujetos humanos: implicancias lógicas, históricas y éticas, en Pautas éticas de investigación en sujetos humanos: nuevas perspectivas, Lolas F, Quezada A. ed. Programa regional de Bioética OPS, OMS, 2003.

2.- CIOMS 2002. Pautas éticas internacionales para la investigación biomédica en seres humanos, Ginebra 2002, 20.

3.- EMMANUEL E. ¿Qué hace que la investigación clínica sea ética? Siete requisitos éticos, en Pautas éticas de investigación en sujetos humanos: nuevas perspectivas, Lolas F y Quezada A ed. Programa regional de bioética OPS, OMS, 2003.
Correspondencia a:

Prof. Marianne Gaudlitz Harnecker

Departamento de Bioética

Facultad de Medicina

Universidad de Chile

Independencia 1027, Piso 4. Santiago.

E-mail: m.gaudlitz@entelchile.net 